\title{
Identificación de factores de predicción del incumplimiento terapéutico en adultos mayores hipertensos de una comunidad del sur de Chile
}

\author{
Sara Mendoza-Parra, ${ }^{1}$ José Manuel Merino ${ }^{2}$ y Omar A. Barriga ${ }^{2}$
}

Forma de citar

Mendoza-Parra S, Merino JM, Barriga OA. Identificación de factores de predicción del incumplimiento terapéutico en adultos mayores hipertensos de una comunidad del sur de Chile. Rev Panam Salud Publica. 2009;25(2):105-12.

RESUMEN Objetivo. Identificar factores de predicción del incumplimiento terapéutico en adultos mayores hipertensos a partir de su dependencia funcional, trastornos de memoria, percepción de bienestar, maltrato y depresión en una región del sur de Chile.

Métodos. Estudio cuantitativo exploratorio en una muestra de 211 adultos mayores hipertensos (29,1\% de los atendidos en el Programa Cardiovascular del Centro de Salud Familiar San Pedro, en la provincia de Concepción, Región del Bío Bio, Chile). Se aplicaron siete instrumentos: el cuestionario abreviado de Pfeiffer para el estado mental, la escala de Yesavage para la depresión geriátrica, la escala de maltrato senil, la escala moral del Centro Geriátrico de Filadelfia, la escala de conductas en salud y las escalas de Lawton y Katz para medir la capacidad de realizar actividades instrumentales y básicas de la vida diaria, respectivamente. Mediante el análisis de componentes principales se definieron variables latentes.

Resultados. Se determinaron dos variables latentes: vinculación con el medio - medida a partir de las variables depresión, maltrato y satisfacción con el medio-y autonomía - medida a partir de las variables estado mental y capacidad para realizar actividades instrumentales y básicas-. Estas variables latentes explicaron 39,7\% y 20,7\% del incumplimiento terapéutico, respectivamente.

Conclusiones. Las variables latentes propuestas pueden emplearse como factores de predicción del incumplimiento terapéutico de los adultos mayores con hipertensión arterial. Las causas del incumplimiento terapéutico no pueden medirse solamente en los establecimientos de salud, es necesario conocer el entorno primario en el hogar y adecuar la atención sanitaria a partir de las necesidades que allí se detecten.

Palabras clave Negativa del paciente al tratamiento, hipertensión, factores de riesgo, salud del anciano, Chile.

Departamento de Enfermería, Facultad de Medicina, Universidad de Concepción, Concepción, Chile. La correspondencia se debe dirigir a Sara Mendoza-Parra, Departamento de Enfermería, Facultad de Medicina, Universidad de Concepción, Casilla 160 C, Concepción, Chile. Correo electrónico: smendoza@udec.cl

2 Departamento de Sociología, Facultad de Ciencias Sociales, Universidad de Concepción, Concepción, Chile.
La mortalidad por hipertensión arterial (HTA) aumenta bruscamente con la edad en la población de adultos mayores: según cifras del año 1996, en Chile pasó de 41,9 por 100000 habitantes en el grupo de 65 a 69 años a 443,2 por 100000 habitantes en el de 80 años o más (1). Un reciente estudio de los adultos mayores beneficiarios del sistema público de salud encontró que en la Región del Bío Bío - la tercera región chilena más envejecida de las 15 que componen el país-, $62 \%$ de la prevalencia total de enfermedades crónicas corresponde a la HTA, con valores elevados de depresión moderada $(25,3 \%)$ e intensa $(9,7 \%)$. Además, 
se observó que muchos de los adultos mayores sufrían diferentes formas de maltrato físico, psicológico y social (12\%) y tenían un bajo grado de adaptación social $(40 \%)$, deterioro leve o intenso de la memoria $(49 \%)$ y dependencia total $(1,4 \%)(2)$. Las cifras de dependencia funcional fueron similares a las observadas en estudios realizados en comunas de la parte central del país —donde se encuentra el Bío Bío (3) - y en otros países, como Cuba (4). Por su parte, la frecuencia de depresión mostró cifras similares a las encontradas recientemente en el Área Metropolitana, donde se ubica la capital del país (5). Asimismo, el maltrato duplicaba la media nacional (2-5\%) y la media mundial de maltrato doméstico e institucional (4-6\%) al anciano (6).

Ante esta cruda realidad local y el hecho de que el cumplimiento de los tratamientos por parte de los adultos mayores podría verse afectado por sus condiciones de vida, es vital determinar la relación entre las circunstancias de mayor dependencia funcional, el maltrato, la falta de memoria, los bajos niveles de adaptación y la depresión de los ancianos, por una parte, y el incumplimiento terapéutico, por la otra.

En los últimos años se ha observado un crecimiento sustancial en el número de artículos científicos dedicados a este tema, lo que refleja el esfuerzo que se realiza por identificar los factores que pueden estar influyendo en la decisión de los pacientes de no seguir las recomendaciones médicas. Pero este fenómeno se ha explorado menos en los adultos mayores o se ha enfocado casi exclusivamente en aspectos directamente relacionados con el control de la enfermedad (ingesta de medicamentos, relación médico-paciente, organización sanitaria, conocimiento de la enfermedad, calidad de la atención, etc.). Por ello aún queda pendiente explorar las circunstancias de la vida que pudieran servir como indicadores concretos de la predisposición de los adultos mayores a adoptar conductas inadecuadas, tales como el incumplimiento del tratamiento para alguna enfermedad crónica.

Esta realidad, aún no mostrada en su magnitud real, podría estar relacionada con los sentimientos insuperables de desesperanza que siente una parte de los adultos mayores y la incapacidad de cuidarse a sí mismos o seguir las indicaciones médicas. A lo anterior se debe añadir el deterioro funcional y cognitivo que acompaña al envejecimiento, lo que unido a lo anterior podría ayudar a conformar un perfil característico que permita predecir la propensión al incumplimiento terapéutico en este sensible grupo de la población.

El presente estudio tuvo como objetivo identificar factores de predicción del incumplimiento terapéutico en adultos mayores hipertensos a partir de su dependencia funcional, trastornos de memoria, percepción de bienestar, maltrato y depresión en una región del sur de Chile.

\section{MATERIALES Y MÉTODOS}

Se realizó un estudio cuantitativo exploratorio entre los meses de junio de 2004 y enero de 2005 en una población de 725 adultos mayores hipertensos participantes en el Programa Cardiovascular del Centro de Salud Familiar San Pedro. Este establecimiento del nivel primario de atención perteneciente a la red pública de salud está ubicado en la comuna San Pedro de la Paz, provincia de Concepción, Región del Bío Bío, Chile. Los criterios de inclusión fueron tener 65 años o más y diagnóstico de HTA con indicación de tratamiento farmacológico, no presentar otras enfermedades crónicas, residir en el área de influencia del centro de salud y haber dado su consentimiento informado para participar en esta investigación.

Mediante un muestreo aleatorio sistemático se seleccionó uno de cada tres pacientes consecutivos hasta conformar una muestra de 245 personas. Debido a la necesidad de tener mediciones homogéneas se excluyeron de la muestra a las personas que fumaban o tenían un trabajo remunerado, por lo que la muestra definitiva quedó constituida por 211 personas (29,1\% de la población de partida).

\section{Variables e instrumentos utilizados}

Los antecedentes sociodemográficos, de salud e indicaciones terapéuticas se tomaron de la documentación clínica que se mantiene de cada paciente en el Programa Cardiovascular y se realizó una visita domiciliaria en la que se aplicaron los siguientes instrumentos:

Cuestionario abreviado del estado mental, de Pfeiffer (7). Esta es la escala más utilizada en español para valorar el deterioro cognitivo por su brevedad, facilidad de ejecución, validez (sensibilidad:
$68 \%$; especificidad: $96 \%$; valor predictivo positivo: $92 \%$; y valor predictivo negativo: $82 \%$ ) y buena reproducibilidad $(0,82-0,83)(8)$. Consta de 10 preguntas sobre orientación, memoria y cálculos sencillos. La suma de las puntuaciones por respuesta (incorrecto: 1; correcto: 0) permite clasificar a las personas en cuatro grupos: normal (0-2 puntos), deterioro leve (3-4 puntos), deterioro moderado (5-7 puntos) y deterioro intenso (8-10 puntos) (9).

Escala de depresión geriátrica, de Yesavage (10). Se utilizó la versión reducida adaptada y validada al castellano con resultados similares a los del cuestionario original (sensibilidad: $81,1 \%$; especificidad: $76,7 \%$; fiabilidad en un mismo observador: 0,95; fiabilidad entre observadores: 0,65; y consistencia interna: 0,99) (11). Consta de 15 preguntas que indagan sobre síntomas de trastornos psiquiátricos. La suma de las puntuaciones por respuesta (positiva: 1; negativa: 0 ) permite clasificar a las personas en tres categorías: sin depresión (0-5 puntos), depresión moderada (6-9 puntos) y depresión intensa (más de 10 puntos) (9).

Escala de Lawton (12). Mide la capacidad para realizar actividades instrumentales de la vida diaria (AIVD). Estudios en la población española han permitido concluir que es una herramienta válida $\mathrm{y}$ confiable para complementar la valoración cognitiva (consistencia interna: 0,78; fiabilidad dentro de una misma clase: $0,95)$, pues añade poder predictivo a las pruebas breves que miden el estado mental (13). Explora ocho actividades (usar el teléfono, ir de compras, preparar comida, cuidar la casa, lavar la ropa, utilizar medios de transporte, cumplir la medicación y utilizar dinero). La suma de las puntuaciones por respuesta (puede realizar la actividad: 1 ; no puede realizarla: 0 ) permite clasificar a las personas en tres categorías: independencia total (8 puntos), dependencia moderada (4-7 puntos) y dependencia intensa (0-3 puntos) (9).

Escala de Katz (14). Mide la capacidad de realizar actividades básicas de la vida diaria (ABVD), como bañarse, moverse, vestirse, controlar los esfínteres, ir al baño y comer. La suma de las puntuaciones por respuesta (puede realizar la actividad: 1 ; no puede realizarla: 0 ) permite clasificar a las personas en siete grupos: A para las personas con independencia 
total, del grupo B al F para los que tienen diferentes grados de dependencia parcial y el $G$ para las personas con dependencia total (9). Esta escala refleja la gama más básica de necesidades de los ancianos en la comunidad y ha sido especialmente útil para que los planificadores de los servicios de salud, profesionales e investigadores diseñen programas de atención dirigidos a los adultos mayores $(15,16)$. A pesar de que este instrumento ha sufrido modificaciones y se han utilizado diferentes enfoques respecto a la puntuación, su utilidad para evaluar el estado funcional en la población de adultos mayores ha quedado demostrada siempre (17) y se ha confirmado su confiabilidad y validez en diferentes grupos étnicos (18).

Escala de maltrato senil (19). Evalúa todo acto con intención de dañar al adulto mayor perpetrado por uno o varios de sus familiares. Indaga sobre el posible maltrato físico o psicológico y pregunta si el anciano ha recibido golpes o empujones, si ha sido objeto de amenazas o chantajes, si ha recibido insultos o burlas y si es considerado en la toma de decisiones familiares. Su aplicación en adultos mayores peruanos mostró una elevada consistencia interna (coeficiente alfa de Cronbach: 0,798).

Escala moral del Centro Geriátrico de Filadelfia $(2,20)$. Mide el grado subjetivo de satisfacción o bienestar del anciano con su situación actual en tres sentidos: actitud hacia el propio envejecimiento, insatisfacción con la soledad, y ansiedad o inquietud. Consta de 17 preguntas. La suma de las puntuaciones por respuesta (satisfacción o bienestar positivo: 1 ; negativo: 0 ) permite clasificar a las personas en tres categorías según su grado de bienestar: alto (13-17 puntos), mediano (9-12 puntos) y bajo (0-8 puntos). Esta evaluación refleja el grado de adaptación del adulto mayor.

Escala de conductas en salud. Se utilizó la escala modificada (21), que permite caracterizar la adhesión al tratamiento. La suma de las puntuaciones por respuesta (nunca: 1 ; a veces: 2 ; siempre: 3 ) permite alcanzar de 12 a 36 puntos. Cuatro subescalas indagan sobre la dieta, las actividades que realizan, si toman los medicamentos y si pueden controlar situaciones de estrés, en cada uno de los contextos en que se desarrolla la vida diaria del adulto mayor (el hogar, los deportes o la recrea- ción, y las actividades sociales). La escala original (22) tiene un formato Likert de cinco alternativas de respuesta que permite alcanzar un máximo de 100 puntos y cuenta con una quinta subescala que indaga sobre el hábito de fumar y se extiende al ámbito laboral. En este estudio se prescindió de estos elementos por no constituir conductas características en la muestra estudiada.

El control de la calidad de los datos de las escalas y las puntuaciones de las respuestas demostraron una aceptable consistencia interna, con valores del coeficiente alfa de Cronbach entre 0,70 y 0,85 , excepto para la escala de maltrato senil que fue de 0,526.

\section{Análisis de los resultados}

Mediante la técnica de análisis de componentes principales (23) se identificaron las variables latentes que pudieran formarse a partir de las correlaciones de las puntuaciones de los diferentes instrumentos utilizados. Las variables latentes deben ser independientes entre sí, tener una elevada correlación con las variables originales y deben poder explicar la varianza de cada componente (24). Se aplicó la rotación denominada varimax para lograr una redistribución de la varianza y un patrón de componentes con mayor poder de discriminación de las variables. Este análisis permite establecer las asociaciones entre cada variable original y las variables latentes propuestas, lo que ayuda a separar e interpretar más claramente los componentes por las variables que lo integran.

Para determinar las variables que pudieran predecir el incumplimiento terapéutico se buscaron asociaciones entre las nuevas variables latentes (variables independientes) y el incumplimiento terapéutico (variable dependiente) mediante la prueba de correlación de Pearson.

\section{RESULTADOS}

\section{Perfil de la muestra}

De los 211 adultos mayores que finalmente formaron parte de la muestra estudiada, $157(74,4 \%)$ eran mujeres y solo $54(25,6 \%)$ eran hombres; la media de la edad fue de 75,4 años (mínima: 65; máxima: 95) y el grupo de 91-95 años estuvo compuesto solo por mujeres.

En el cuadro 1 se muestran las características sociodemográficas y de la enfer- medad hipertensiva más importantes del grupo de estudio. El estado civil más frecuente fue el de casado o conviviente, con $102(48,3 \%)$ participantes, mientras que el nivel educacional que predominó fue el básico con 139 (65,9\%) personas. De los participantes, $132(62,6 \%)$ profesaban la religión católica.

En total, 145 (68,7\%) de los participantes presentaban HTA en etapa 1 y 157 $(74,4 \%)$ se clasificaron con riesgo cardiovascular moderado; solo $56(26,5 \%)$ no aceptaban que su enfermedad era para toda la vida y $123(58,3 \%)$ no se sentían enfermos (cuadro 1).

Como resultado de las encuestas se encontró que solamente $9(4,3 \%)$ de los adultos mayores estudiados tenían dependencia total para realizar AIVD y solo una mujer $(0,5 \%)$ presentaba dependencia total para las ABVD. De los participantes, $40(19,0 \%)$ presentaban deterioro intelectual moderado y $9(4,3 \%)$ intenso; del total de los encuestados, solo $8(3,8 \%)$, todas mujeres, manifestaron haber sufrido maltrato físico, $24(11,4 \%)$ habían sido insultados; 7 (3,3\%) habían recibido amenazas o chantaje, y entre $11,4 \%$ y $24,2 \%$ afirmaron que no conversaban con ellos, no se sentían queridos o no eran consultados para la toma de decisiones familiares. En total, $78(37,0 \%)$ presentaron depresión moderada y 21 (10,0\%) depresión intensa (cuadro 2).

\section{Factores de predicción del incumplimiento}

Se observó una relación altamente significativa $(P<0,02)$ entre la mayoría de las escalas entre sí, excepto entre el cuestionario de Pfeiffer y la escala moral $(P=0,102)$.

A partir de la correlación existente entre las escalas, se observó un primer grupo conformado por las variables de depresión (según la escala de depresión geriátrica), maltrato (según la escala de maltrato senil) y la percepción de bienestar (según la escala moral) y un segundo grupo conformado por las variables que reflejaban el estado mental (según el cuestionario de Pfeiffer), la dependencia para realizar AIVD (según la escala de Lawton) y la dependencia para realizar ABVD (según la escala de Katz). Si bien el primer grupo de variables da cuenta de aspectos que tienen que ver con la vinculación social del adulto mayor (depresión, maltrato y percepción de bienestar), el segundo grupo reúne varia- 
CUADRO 1. Características sociodemográficas y de la enfermedad hipertensiva de la muestra estudiada. Programa Cardiovascular del Centro de Salud Familiar San Pedro, Concepción, Chile, junio 2004-enero 2005

\begin{tabular}{|c|c|c|c|c|c|c|}
\hline \multirow[b]{2}{*}{ Característica } & \multicolumn{2}{|c|}{ Mujeres } & \multicolumn{2}{|c|}{ Hombres } & \multicolumn{2}{|c|}{ Total } \\
\hline & No. & $\%$ & No. & $\%$ & No. & $\%$ \\
\hline \multicolumn{7}{|l|}{ Estado civil } \\
\hline Con pareja (casado/a o conviviente) & 59 & 28,0 & 43 & 20,4 & 102 & 48,3 \\
\hline Sin pareja (separado/a, anulado/a) ${ }^{a}$ & 30 & 14,2 & 4 & 1,9 & 34 & 16,1 \\
\hline Viudo/a & 68 & 32,2 & 7 & 3,3 & 75 & 35,5 \\
\hline \multicolumn{7}{|l|}{ Nivel educacional } \\
\hline Sin instrucción formal & 20 & 9,5 & 3 & 1,4 & 23 & 10,9 \\
\hline Básica (8 años de estudio) & 107 & 50,7 & 32 & 15,2 & 139 & 65,9 \\
\hline Media (12 años de estudio) & 25 & 11,8 & 16 & 7,6 & 41 & 19,4 \\
\hline Superior (universitario) & 5 & 2,4 & 3 & 1,4 & 8 & 3,8 \\
\hline \multicolumn{7}{|l|}{ Religión } \\
\hline Católica & 96 & 45,5 & 36 & 17,1 & 132 & 62,6 \\
\hline Evangélica & 47 & 22,3 & 13 & 6,2 & 60 & 28,4 \\
\hline Otras (judaica, mormona, ortodoxa, etc.) & 9 & 4,3 & 2 & 0,9 & 11 & 5,2 \\
\hline Sin religión & 5 & 2,4 & 3 & 1,4 & 8 & 3,8 \\
\hline \multicolumn{7}{|l|}{ Tipo de hipertensión arterial } \\
\hline Etapa $1^{\mathrm{b}}$ & 109 & 51,7 & 36 & 17,1 & 145 & 68,7 \\
\hline Etapa $2^{c}$ & 37 & 17,5 & 16 & 7,6 & 53 & 25,1 \\
\hline Etapa $3^{d}$ & 11 & 5,2 & 2 & 0,9 & 13 & 6,2 \\
\hline \multicolumn{7}{|l|}{ Tipo de riesgo cardiovascular } \\
\hline Moderado & 115 & 54,5 & 42 & 19,9 & 157 & 74,4 \\
\hline Alto & 37 & 17,5 & 12 & 5,7 & 49 & 23,2 \\
\hline Máximo & 5 & 2,4 & 0 & 0,0 & 5 & 2,4 \\
\hline \multicolumn{7}{|l|}{ Acepta la enfermedad } \\
\hline $\mathrm{Si}$ & 116 & 55,0 & 39 & 18,5 & 155 & 73,5 \\
\hline No la acepta o no lo ha pensado & 41 & 19,4 & 15 & 7,1 & 56 & 26,5 \\
\hline \multicolumn{7}{|l|}{ Percepción de la enfermedad } \\
\hline Se siente enfermo/a & 71 & 33,6 & 17 & 8,1 & 88 & 41,7 \\
\hline No se siente enfermo/a & 86 & 40,8 & 37 & 17,5 & 123 & 58,3 \\
\hline Total & 157 & 74,4 & 54 & 25,6 & 211 & 100,0 \\
\hline
\end{tabular}

a En Chile se aprobó el divorcio en 2004. Las clasificaciones de separado o anulado son parte de las categorías oficiales legales utilizadas por el INE para caracterizar el estado civil en el país.

b Etapa 1 de la hipertensión arterial: pacientes con tensión sistólica entre 149 y $159 \mathrm{~mm} \mathrm{Hg}$ y diastólica entre 90 y $99 \mathrm{~mm} \mathrm{Hg}$.

c Etapa 2 de la hipertensión arterial: pacientes con tensión sistólica entre 160 y 179 mm Hg y diastólica entre 100 y 109 mm Hg.

d Etapa 3 de la hipertensión arterial: pacientes con tensión sistólica por encima de $179 \mathrm{~mm} \mathrm{Hg}$ y diastólica superior a $109 \mathrm{~mm} \mathrm{Hg}$.

bles relacionadas con la capacidad de desenvolverse autónomamente en ese medio (capacidades intelectual y funcional). De esta manera, de las seis variables originales emergieron dos nuevas dimensiones o variables latentes, denominadas vinculación con el medio y autonomía, capaces de explicar en conjunto $60,4 \%$ de la varianza observada entre las variables analizadas $(39,7 \%$ por la variable latente vinculación con el medio y $20,7 \%$ por la variable latente autonomía).

Las variables vinculadas entre sí en una matriz de relaciones mutuas se incorporaron por separado debido a la calidad de la representación que adquieren en las nuevas dimensiones extraídas. Así, los valores de carga más alta de depresión, maltrato y percepción de bienestar incorporadas a la primera dimensión o variable latente $(0,839,0,637$ y $-0,852$, respectivamente) indicaron que esos componentes estaban mejor representados en la dimensión vinculación con el medio (cuadro 3 ). Algo similar se observó con respecto a las variables relacionadas con las capacidades intelectuales y funcionales incorporadas a la nueva dimensión autonomía. Los valores positivos de depresión y maltrato indican que se correlacionaron positivamente con la nueva dimensión, es decir, a mayor puntuación en las escalas de depresión y maltrato, la variable latente vinculación con el medio aumentaría su valor, en cambio a mayor puntuación en la escala que mide la percepción de bienestar, la nueva variable sería menor. Con la variable latente autonomía ocurriría lo contrario: a mayor dependencia para realizar ABVD, la dimensión latente adquiriría menor valor.

La consistencia interna alcanzada por las escalas en cada nueva dimensión fueron aceptables $(0,70$ para la vinculación con el medio y 0,63 para la autonomía), por lo que se pueden utilizar para evaluar el cumplimiento terapéutico de estos pacientes.

Por tanto, a partir de los promedios de las variables relacionadas con la de- presión, el maltrato y el estado mental (según las escalas correspondientes) se puede determinar el valor de la variable latente vinculación con el medio como primer factor de predicción de la no adhesión al tratamiento, y a partir de los promedios de las variables relacionadas con el estado mental y la dependencia para realizar AIVD y ABVD (según las respectivas escalas) se puede establecer el valor de la variable latente autonomía, un segundo factor de predicción del incumplimiento terapéutico. Esto quedó confirmado por los valores altamente significativos de la correlación entre estas nuevas variables latentes y la no adhesión al tratamiento (cuadro 4).

\section{DISCUSIÓN}

La mayor proporción de mujeres en el grupo de adultos mayores le imprime a la carga de morbimortalidad por HTA las características propias de la situación de salud de las mujeres chilenas de este grupo de edad, que por ser viudas o solteras tienen menos posibilidades de apoyo cercano para tratar su enfermedad y que por tener un bajo nivel de escolaridad pueden tener dificultades para tomar decisiones acertadas relacionadas con su salud o para movilizar recursos y redes comunitarias. Estos resultados coinciden con lo encontrado por Mendoza y colaboradores en 2004 (25) en el sentido de que ser mujer, viuda, vivir sola y ser pobre eran factores asociados no solo con la mayor prevalencia de HTA, sino también con el menor cumplimiento terapéutico. Esto podría indicar que, al menos en Chile, las mujeres adultas mayores presentan con mayor frecuencia que los hombres los tres problemas de mayor impacto sobre los ancianos: peor salud, soledad y pobreza (26).

Llama la atención la baja prevalencia de la dependencia total para realizar ABVD en el grupo estudiado $(0,5 \%)$ en comparación con la prevalencia nacional $(3,5 \%)(1)$. Esto puede deberse a la exclusión en este estudio de los adultos mayores con enfermedades adicionales a la HTA, los que presentarían un mayor deterioro y por lo tanto una mayor dependencia funcional. Se comprobó también que a pesar de tener un diagnóstico de HTA, una gran parte de estos pacientes no presentaba síntomas de la enfermedad $(58,3 \%)$ y no consideraba que esta es una enfermedad con la que vivirá toda la vida $(26,6 \%)$, lo que ha sido relacionado 
CUADRO 2. Características de la muestra estudiada a partir de las respuestas a las encuestas. Programa Cardiovascular del Centro de Salud Familiar San Pedro, Concepción, Chile, junio 2004enero 2005

\begin{tabular}{|c|c|c|c|c|c|c|}
\hline \multirow[b]{2}{*}{ Variables } & \multicolumn{2}{|c|}{ Mujeres } & \multicolumn{2}{|c|}{ Hombres } & \multicolumn{2}{|c|}{ Total } \\
\hline & No. & $\%$ & No. & $\%$ & No. & $\%$ \\
\hline \multicolumn{7}{|l|}{ Dependencia para realizar $A B V D^{a}$} \\
\hline Independencia total & 131 & 62,1 & 49 & 23,2 & 180 & 85,3 \\
\hline Dependencia parcial & 25 & 11,8 & 5 & 2,4 & 31 & 14,2 \\
\hline Dependencia total & 1 & 0,5 & 0 & 0,0 & 1 & 0,5 \\
\hline \multicolumn{7}{|l|}{ Dependencia para realizar AIVD } \\
\hline Independencia total & 61 & 28,9 & 21 & 10,0 & 82 & 38,9 \\
\hline Dependencia moderada & 88 & 41,7 & 32 & 15,2 & 120 & 56,9 \\
\hline Dependencia intensa & 8 & 3,8 & 1 & 0,5 & 9 & 4,3 \\
\hline \multicolumn{7}{|l|}{ Depresiónc ${ }^{c}$} \\
\hline Sin depresión & 79 & 37,4 & 33 & 15,6 & 112 & 53,1 \\
\hline Depresión moderada & 61 & 28,9 & 17 & 8,1 & 78 & 37,0 \\
\hline Depresión intensa & 17 & 8,1 & 4 & 1,9 & 21 & 10,0 \\
\hline \multicolumn{7}{|l|}{ Grado de bienestar (estado moral) $^{d}$} \\
\hline Alto & 36 & 17,1 & 16 & 7,6 & 52 & 24,6 \\
\hline Mediano & 66 & 31,3 & 26 & 12,3 & 92 & 43,6 \\
\hline Bajo & 55 & 26,1 & 12 & 5,7 & 67 & 31,8 \\
\hline \multicolumn{7}{|l|}{ Refiere maltrato ${ }^{\mathrm{e}}$} \\
\hline Golpes o empujones & 8 & 3,8 & 0 & 0,0 & 8 & 3,8 \\
\hline Insultos o gritos & 17 & 8,1 & 7 & 3,3 & 24 & 11,4 \\
\hline Amenazas o chantaje & 6 & 2,8 & 1 & 0,5 & 7 & 3,3 \\
\hline No lo consideran en la toma de decisiones & 42 & 19,9 & 9 & 4,3 & 51 & 24,2 \\
\hline No se siente querido & 17 & 8,1 & 4 & 1,9 & 21 & 10,0 \\
\hline No conversan con ellos & 19 & 9,0 & 5 & 2,4 & 24 & 11,4 \\
\hline \multicolumn{7}{|l|}{ Estado mental ${ }^{\dagger}$} \\
\hline Normal & 71 & 33,6 & 30 & 14,2 & 101 & 47,9 \\
\hline Deterioro leve & 45 & 21,3 & 16 & 7,6 & 61 & 28,9 \\
\hline Deterioro moderado & 33 & 15,6 & 7 & 3,3 & 40 & 19,0 \\
\hline Deterioro intenso & 8 & 3,8 & 1 & 0,5 & 9 & 4,3 \\
\hline \multicolumn{7}{|l|}{ Adhesión al tratamiento ${ }^{g}$} \\
\hline Cumple & 29 & 13,7 & 19 & 9,0 & 48 & 22,7 \\
\hline No cumple & 128 & 60,7 & 35 & 16,6 & 163 & 77,3 \\
\hline Total & 157 & 74,4 & 54 & 25,6 & 211 & 100,0 \\
\hline
\end{tabular}

a ABVD: actividades básicas de la vida diaria, según la escala de Katz (14).

${ }^{b}$ AIVD: actividades instrumentales de la vida diaria, según la escala de Lawton (12).

c Según la escala de depresión geriátrica, de Yesavage (10).

d Según la escala moral del Centro Geriátrico de Filadelfia $(2,20)$.

e Según la escala de maltrato senil (19).

Según el cuestionario abreviado del estado mental, de Pfeiffer (7)

g Según la escala de conductas en salud (21).

directamente con el incumplimiento terapéutico $(21,25,27)$. A pesar de que el maltrato es una condición que muchos entrevistados se niegan a reconocer, solo 7 de las 211 personas estudiadas no informaron maltratos en ninguna de las situaciones planteadas. Todos los casos de maltrato físico se encontraron en mujeres, incluso en una persona de 81 años (cuadro 2).

Debido a la relativamente baja consistencia interna de la escala de maltrato en este estudio (alfa de Cronbach: 0,526), se podría cuestionar la inclusión de este indicador en la exploración realizada, sin embargo esta fue una decisión acertada, ya que tuvo el comportamiento esperado como parte de la variable latente vinculación con el medio.

Por el comportamiento que tuvieron las diferentes escalas en la exploración de nuevas dimensiones explicativas del incumplimiento terapéutico se puede constatar que tanto el ámbito social como la capacidad del adulto mayor para desenvolverse en él pueden ser factores de predicción de ese incumplimiento. Lo anterior confirma la necesidad de que los equipos de salud dejen de reducir la atención sanitaria de los adultos mayores a los términos del envejecimiento biológico y comiencen a considerar elementos clave de su salud, como el género, la clase social, el nivel educacional y las condiciones de vida (28).

En la actualidad hay consenso en que conforme ha aumentado la proporción de personas de edad avanzada que no cuentan con suficientes recursos y que sufren las desigualdades de la atención sanitaria, también ha aumentado el número que padece discapacidades y que sufre los efectos negativos tanto del aislamiento físico como del emocional. El mayor riesgo de deterioro en la calidad de vida lo presentan las personas que no cuentan con apoyo familiar (29). La necesidad de compañía y de ser interpretados según sus convicciones, valores y sentimientos influye positivamente en la etapa final de la vida de los ancianos (30), mientras que vivir marginado no favorece una vejez sana. El aislamiento y la soledad impiden que los adultos mayores mantengan relaciones sociales efectivas, exploren su capacidad de cambio y se sientan partícipes de su entorno social e integrados a él.

Las escalas reunidas en la variable latente vinculación con el medio son claramente indicativas de lo anterior, es decir, del impacto que produce la falta de interacción de los ancianos con su medio social inmediato. La escala moral empleada, por ejemplo, indaga sobre aspectos relacionados con la satisfacción de la persona en la vejez, en lo útil que se siente a la edad que tiene, la frecuencia con que es visitado por amigos, etc., cuestiones todas relacionadas con la forma en que se desarrolla su vida como "anciano" y el entorno que rodea su vida cotidiana. Todo esto influye en sus decisiones de salud, sobre todo si debe enfrentar una enfermedad como la HTA, que impone drásticos cambios en sus hábitos. La escala de depresión, por su parte, indaga sobre aspectos directamente relacionados con la estimación subjetiva que la persona siente respecto a su estado de ánimo, felicidad, utilidad y satisfacción con la vida, elementos cruciales a la hora de involucrarse en nuevas actividades, para tratar la enfermedad o asumir acciones preventivas ante nuevos riesgos derivados del propio envejecimiento una vez que presenta la HTA.

El estudio integral de los adultos mayores hipertensos es de suma importancia al ingresar a los programas de atención, pues —al menos en este grupoel entorno familiar es el factor que más directamente influye en su adhesión a las recomendaciones terapéuticas, especialmente cuando el tratamiento es autoadministrado (31). Poder aplicar instrumentos breves que indaguen aspectos como los descritos, es decir, situaciones de violencia, abuso doméstico y síntomas de depresión, puede ayudar a detectar a los ancianos en riesgo de no cumplir las recomendaciones a corto y mediano plazos. Si se observa, entre 
CUADRO 3. Índices de correlación entre las variables originales y las variables latentes extraídas mediante la rotación ortogonal varimax

\begin{tabular}{|c|c|c|}
\hline \multirow[b]{2}{*}{ Variables originales } & \multicolumn{2}{|c|}{ Variables latentes } \\
\hline & Vinculación con el medio & Autonomía \\
\hline Dependencia para realizar AIVDa & $-0,106$ & 0,838 \\
\hline Depresión & 0,839 & $-0,198$ \\
\hline Percepción de bienestar ${ }^{c}$ & $-0,852$ & 0,026 \\
\hline Refiere maltrato $^{d}$ & 0,637 & $-0,168$ \\
\hline Estado mentale & $-0,102$ & 0,711 \\
\hline Dependencia para realizar ABVD ${ }^{f}$ & 0,161 & $-0,682$ \\
\hline
\end{tabular}

a AIVD: actividades instrumentales de la vida diaria, según la escala de Lawton (12).

b Según la escala de depresión geriátrica, de Yesavage (10).

c Según la escala moral del Centro Geriátrico de Filadelfia $(2,20)$.

d Según la escala de maltrato senil (19).

e Según el cuestionario abreviado del estado mental, de Pfeiffer (7).

† ABVD: actividades básicas de la vida diaria, según la escala de Katz (14).

CUADRO 4. Correlación entre las variables latentes vinculación con el medio y autonomía entre sí y con el incumplimiento terapéutico

\begin{tabular}{|c|c|c|c|}
\hline Variable & $\begin{array}{l}\text { Incumplimiento } \\
\text { terapéutico }\end{array}$ & $\begin{array}{l}\text { Vinculación con } \\
\text { el medio }\end{array}$ & Autonomía \\
\hline Incumplimiento terapéuticob & 1,000 & & \\
\hline Vinculación con el mediob & 0,316 & 1,000 & \\
\hline Autonomía & 0,155 & 0,318 & 1,000 \\
\hline Incumplimiento terapéutico ${ }^{C}$ & & $<0,001$ & 0,012 \\
\hline Vinculación con el medio ${ }^{C}$ & $<0,001$ & & $<0,001$ \\
\hline Autonomía & 0,012 & $<0,001^{a}$ & \\
\hline
\end{tabular}

a Según la escala de conductas en salud, modificada (21).

b Según la prueba de correlación de Pearson. Valor de significación $P \leq 0,05$

c Según la prueba de significación unilateral. Valor de significación $P \leq 0,05$.

otros signos, que un anciano concurre frecuentemente a los servicios de urgencia porque una afección crónica ha empeorado a pesar de tener a su disposición las medidas terapéuticas para controlarla, se debe investigar la posibilidad de que sea víctima de maltrato (29). Lo anterior, a la vez que es una clara consecuencia del incumplimiento terapéutico, es también un ejemplo de las consecuencias negativas que puede tener el maltrato para el control de las enfermedades crónicas en los adultos mayores.

Además, ante la sospecha de deterioro funcional o intelectual, que fueron las variables que primaron en la variable latente relacionada con la autonomía, los equipos de salud deberían adoptar modalidades de control de la enfermedad en el domicilio y constatar las dificultades que los ancianos tienen para seguir las recomendaciones indicadas en su tratamiento. Perder las habilidades sociales -que abarcan los componentes cognitivos (percepción y procesamiento de información) y conductuales (capacidades funcionales)_ impide que las personas puedan relacionarse de un modo efec- tivo y hace que se pierda la capacidad de seleccionar información relevante y útil, que más tarde puede utilizarse en beneficio de la adopción de una determinada conducta en salud (32), como el adecuado cumplimiento terapéutico.

Si bien para la mayoría de las personas puede ser fácil recordar los horarios, las dosis, el lugar donde se guardan los medicamentos o, simplemente, poder reconocer entre muchos fármacos cuál es el indicado para su enfermedad, para muchos adultos mayores la realidad puede ser muy diferente. En muchos casos, la pérdida de la memoria no les permitirá recordar igual que antes y la mala visión les impedirá distinguir un fármaco de otro, por lo que deberán acudir a un familiar o a la persona más próxima en busca de ayuda. Pero, ¿qué pasa cuando viven solos o qué pueden hacer si, aún sabiendo que deben tomase los medicamentos, no recuerdan el horario o no pueden distinguir el remedio y no quieren pedir ayuda para no sumar preocupaciones al entorno familiar? Es importante considerar lo anterior al recomendar a un adulto mayor hipertenso las indicaciones para tratar su enfermedad, pues la decisión que tome puede estar directamente relacionada con la capacidad de recordar esas indicaciones, de tener el ánimo suficiente para realizar nuevas actividades o de contar con el apoyo familiar necesario. Si vive solo, es de gran importancia la capacidad asertiva que pueda emplear para movilizar las redes sociales de la comunidad en que vive.

El hogar que acoge a un adulto mayor puede llegar a transformarse en el factor que más le dificulta adoptar conductas de salud favorables. Aunque no es discutible que la familia es el ámbito por excelencia para que los acianos vivan en condiciones adecuadas, se ha advertido que también puede ser la principal fuente de agresiones y abusos hacia estas personas (33). Los síntomas depresivos favorecen el desarrollo de la dependencia funcional y por ello es necesario su reconocimiento sistemático durante la evaluación clínica del adulto mayor (34). Además, se debe tener en cuenta que las dificultades psicológicas son más frecuentes en los ancianos maltratados que en sus pares no maltratados y existen síntomas asociados con los casos de maltrato, como la vergüenza, la culpa, el temor, la ansiedad y la negación, entre otros (29). Con frecuencia es el propio paciente el que trata de ofrecer una imagen lúcida e intacta desde el punto de vista intelectual y su deterioro pasa desapercibido por la familia e incluso por el personal sanitario que lo atiende, lo que hace que sus capacidades cognitivas tiendan a ser sobrevaloradas (20).

Este estudio presenta limitaciones. En primer lugar, al no aplicarse la subescala relacionada con el hábito de fumar y la pregunta que valoraba en cada subescala el cumplimiento en el ambiente laboral, se modificaron las puntuaciones totales de la escala original. Se recomienda, por lo tanto, aumentar el número de encuestados para verificar si estas condiciones se deben medir en este grupo de la población, aunque ambas circunstancias sean poco frecuentes en ellos. Además, es muy posible que no se notifiquen todos los casos de maltrato, por lo que se recomienda utilizar mediciones paralelas, indirectas y validadas, con profesionales capacitados en su detección precoz.

A pesar de esas limitaciones se puede concluir que las dos variables latentes propuestas, vinculación con el medio -medida a partir de las escalas de depresión geriátrica, moral y maltrato 
senil—y autonomía —medida mediante el cuestionario abreviado del estado mental y las escalas de Lawton para AIVD y de Katz para ABVD- explicaron $39,7 \%$ y $20,7 \%$, respectivamente, de la varianza del incumplimiento terapéutico. Ambas variables latentes pueden emplearse como factores de predicción del incumplimiento terapéutico en adultos mayores con HTA.

A partir de estos resultados se recomienda a los equipos de salud implementar estrategias que permitan medir la propensión de los adultos mayores al incumplimiento terapéutico y considerar que esto puede ser resultado de la dependencia funcional, el deterioro cognitivo, la depresión, el maltrato y la insatisfacción con el entorno. Para medir estas situaciones se deben utilizar las escalas empleadas en este estudio, ya que son de fácil aplicación, de bajo costo y no requieren un entrenamiento especial.

El personal de salud vinculado con la atención de los adultos mayores debe capacitarse en el análisis del incumplimiento terapéutico, sus diferentes grados y formas de manifestación y definir actividades concretas dirigidas a promover la adhesión adecuada al tratamiento y a prevenir el incumplimiento. Las causas del incumplimiento terapéutico no pueden medirse solamente en los establecimientos de salud, ya que es necesario conocer el entorno primario en el hogar y adecuar la atención sanitaria a partir de las necesidades que allí se detecten.

Se recomienda estudiar las causas del incumplimiento terapéutico en los adultos mayores en una muestra mayor y observar otras variables latentes que pudieran sumarse a la explicación de esta conducta. Esta debe ser una tarea permanente de los equipos de salud, ya que las circunstancias que determinan esta conducta son múltiples. Se deben desarrollar nuevas estrategias de pesquisa y atención, sobre todo en el grupo de adultos mayores que conforman el segmento de mayor edad y en los que se sospeche que viven en un entorno familiar de maltrato, aislamiento o soledad.

Agradecimientos. Los autores agradecen a la Dirección de Investigación de la Universidad de Concepción, Chile, por el apoyo económico para esta investigación y al grupo de enfermeras del Centro de Salud San Pedro por su colaboración y la valiosa información aportada, que permitieron la aplicación de las encuestas y las visitas domiciliarias a los adultos mayores.

\section{REFERENCIAS}

1. Mardones FS. Algunos antecedentes sobre inequidad en la situación de salud del adulto mayor en Chile. Rev Med Chile. 2004;132(7): $865-72$.

2. Muñoz MP, Mendoza PS, Salazar AM. Dependencia funcional y perfil biosicosocial del adulto mayor. Gerokomos. 2004;15(1):18-27.

3. Díaz VT, Díaz IT, Rojas GC, Novogrodsky DR. Evaluación geriátrica en la atención primaria. Rev Med Chile. 2003;131(8):895-901.

4. Carmenaty ID, Soler LO. Evaluación funcional del anciano. Rev Cubana Enfermer. 2002; 18(3):184-8.

5. Marín LPP, Carrasco GM, Cabezas UM, Gac EH, Hoyl MT, Duery DP, et al. Impacto biomédico de los viajes en adultos mayores chilenos. Rev Med Chile. 2004;132(5):573-8.

6. Marín PP, Castro SH. Abuso o maltrato en el adulto mayor. Bol Esc Med. 2000;29(1-2).

7. Pfeiffer E. A short portable mental status questionnaire for the assessment of organic brain deficit in the elderly patients. J Am Geriatr Soc. 1975;23:433-41.

8. Iráizoz I. Valoración geriátrica integral (II): evaluación nutricional y mental del anciano. An Sist Sanit Navar. 1999;22(Supl1):51-69.

9. Sanjoaquín RAC, Fernández AE, Mesa LMP, García-Arilla E. Valoración geriátrica integral. En: Sociedad Española de Geriatría y Gerontología, ed. Tratado de geriatría para residentes. Madrid: International Marketing and Communication; 2007. Pp. 51-69.

10. Yesavage JA, Brink TL, Rose TL. Development and validation of a geriatric depression screening scale: a preliminary report. J Psychiatr Res. 1983;17:37-49.

11. Martínez IJ, Onis VMC, Dueñas HR, Albert CC, Aguayo TC, Luque LR. Versión española del cuestionario de Yesavage abreviado (EDG) para el despistaje de depresión en maMedifam. 2002;12(10):620-30. yores de 65 años: adaptación y validación.

12. Lawton MP, Brody EM. Assessment of older people: self-maintaining and instrumental activities of daily living. Gerontologist. 1969; 9(3):179-86.

13. Olazarán J, Mouronte P, Bermejo F. Validez clínica de dos escalas de actividades instrumentales en la enfermedad de Alzheimer. Neurología. 2005;20(8):385-434.

14. Katz S, Ford AB, Moskowitz RW, Jackson BA, Jaffe MW. Studies of illness in the aged. The index of ADL: a standardized measure of biological and psychosocial function. J Am Med Assoc. 1963;185:914-9.

15. Álvarez M, de Alaiz T, Brun E, Cabañeros JJ, Calzón M, Cosío I, et al. Capacidad funcional de pacientes mayores de 65 años, según el índice de Katz. Fiabilidad del método. Aten Primaria. 1992;10:812-6.

16. Spector WD, Katz S, Murphy JB, Fulton JP. The hierarchical relationship between activities of daily living and instrumental activities of daily living. J Chronic Dis. 1987;40(6): 481-9.

17. Organización Panamericana de la Salud. Guía clínica para la atención primaria a las personas adultas mayores. 4. ${ }^{\text {a }}$ ed. Washington, D.C.: OPS; 2004. Hallado en http://www. almageriatria.org/documentos/guia_clinica/ 01Portada/01Portada.pdf. Acceso el 2 de diciembre de 2008.

18. Reijneveld SA, Spijker J, Dijkshoorn H. Katz's $\mathrm{ADL}$ index assessed functional performance of Turkish, Moroccan, and Dutch elderly. J Clin Epidemiol. 2007;60(4):382-8.

19. Inga JA, Vara AH. Factores asociados a la satisfacción de vida de adultos mayores de 60 años en Lima, Perú. Univ Phsichol (Colombia). 2006;5(3):475-85.
20. Bayó LJ, Fernández AMC, Orfila PF, Dalfó BA, Casajuana BJ, Vila CIMA, et al. Autopercepción de salud y evaluación integral del paciente anciano en un centro de atención primaria. Aten Primaria. 1996;17(4):273-9.

21. Mendoza PS, Figueroa FM. Creencias en salud y adherencia de pacientes hipertensos controlados en centros de salud de la comuna de Concepción, Chile. Cienc Enferm. 1998; 4(1):63-76.

22. Miller P, Wikoff RL, McMahon M, Garret MJ, Ringel K. Indicators of medical regimen adherence for myocardial infarction patients. Nurs Res. 1985;34:268-72.

23. Batista FJM, Martínez AMR. Análisis multivariante: análisis de componentes principales. Barcelona: Hispano-Europea; 1989.

24. Salinas PH, Albornoz VJ, Reyes PA, Erazo BM, Ide VR. Análisis de componentes principales aplicado a variables respecto a la mujer gestante en la Región de las Américas. Rev Chil Obstet Ginecol. 2006;7(1):17-25.

25. Mendoza PS, Muñoz MP, Merino JM, Barriga O. Factores determinantes de cumplimiento terapéutico en adultos mayores hipertensos. Rev Med Chile. 2004;134(1):65-71.

26. Marín LPP, Guzmán JM, Araya GA. Adultos mayores institucionalizados en Chile: ¿cómo saber cuántos son? Rev Med Chile. 2004;132 (7):832-8.

27. Fasce EH, Flores MA, Fasce FV. Prevalencia de síntomas habitualmente asociados a hipertensión arterial en población normotensa e hipertensa. Rev Med Chile. 2002;130(2): 160-6.

28. Gonzalo E, Pasarín MI. La salud de las personas mayores. Gac Sanit. 2004;18(1):69-80.

29. Envejecimiento y salud: un cambio de paradigma. Rev Panam Salud Publica. 2000;7(1): 60-7. 
30. Figueredo FN, Arcias MRC, Díaz PG. Caracterización del adulto mayor en la comunidad. Rev Cubana Enfermer. 2003;19(3).

31. Sabaté E, ed. Adherence to long-term therapies: evidence for action. Geneva: World Health Organization; 2003. (WHO/MNC/ 03.01.)

32 Carneiro RS, Falcone EMO. Um estudo das capacidades e deficiências em habilidades so- ciais na terceira idade. Psicol Estud. 2004;9(1): 119-26.

33. Minayo MCS. Violência contra idosos: relevância para um velho problema. Cad Saude Publica. 2003;19(3):783-91.

34. Ávila-Funes JA, Melano-Carranza E, Payette $\mathrm{H}$, Amieva H. Síntomas depresivos como factor de riesgo de dependencia en adultos mayores. Salud Publica Mex. 2007;49(5):36775.

Manuscrito recibido el 27 de septiembre de 2007. Aceptado para publicación, tras revisión, el 22 de octubre de 2008.

ABSTRACT Objective. To identify the predictive factors associated with therapy nonadherence among hypertensive older adults, based on functional dependency, memory disorders, self-perceived wellbeing, abuse, and depression in an area of southern Chile.

\section{Identifying predictive factors for therapy nonadherence among hypertensive, older adults from a community in southern Chile}

Key words Treatment refusal, hypertension, risk factors, health of the elderly, Chile.
Methods. A quantitative, exploratory study of a sample of 211 hypertensive, older adults (29.1\% of those being treated by the cardiovascular program at the Centro de Salud Familiar San Pedro, in the province of Concepción, del Bío Bío region, Chile). Seven instruments were applied: Pfeiffer's short mental status questionnaire, Yesavage's geriatric depression scale, the elder abuse screening scale, the morale scale of the Philadelphia Geriatric Center, the health behavior scale, the Lawton assessment of instrumental activities of daily living (ADL), and the Katz ADL index. Following an analysis of the important components, latent variables were identified.

Results. Two latent variables were identified: social connectedness-measured by the variables depression, abuse, and social satisfaction-and autonomy-measured by the variables mental health and ability to perform basic and instrumental ADLs. These latent variables accounted for $39.7 \%$ and $20.7 \%$ of nonadherence to therapy, respectively.

Conclusions. The latent variables proposed here can be used as predictive factors for therapy nonadherence in elderly adults with arterial hypertension. Causes of nonadherence to therapy cannot be assessed solely at health care centers; but rather, an understanding of the home environment is needed and treatment should be adapted according to the needs that exist there.

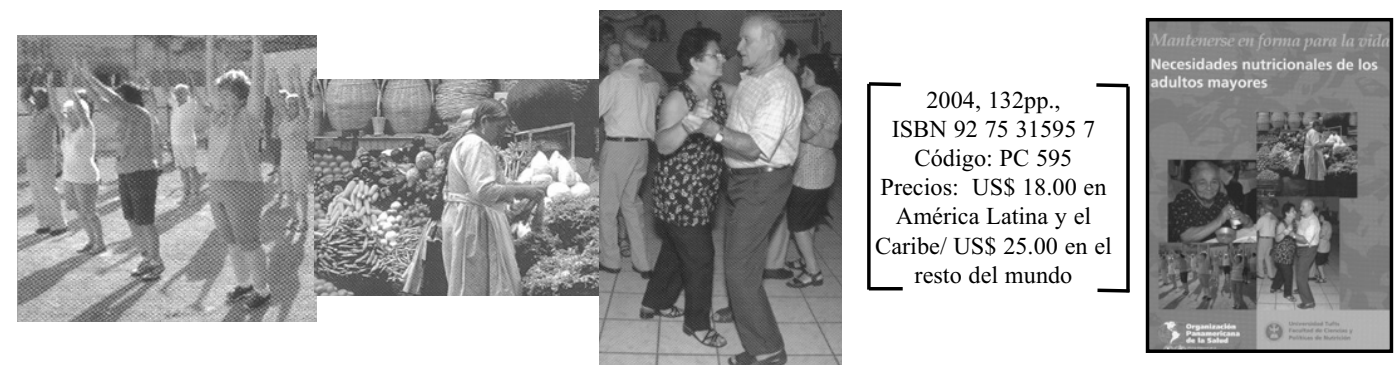

Esta publicación será de mucha utilidad para nutricionistas, médicos generales, gerontólogos, personal de enfermería, proveedores de atención, formuladores de políticas públicas, salubristas y trabajadores sociales. Las recomendaciones específicas sobre la ingesta de nutrientes, el ejercicio y la actividad física también deben interesar a los lectores en general.

Adquiera esta publicación por medio de la librería en línea de la OPS: http://publications.paho.org; correo electrónico: paho@pmds.com 